\title{
Bone formation: Biological aspects and modelling problems
}

\author{
MIGUEL A. HERRERO†* and JOSÉ M. LÓPEZ \\ †Departamento de Matemática Aplicada, Facultad de Matemáticas, Universidad Complutense, 28040 Madrid, Spain \\ ¥Departamento de Morfología y Biología Celular, Facultad de Medicina, IUOPA, Universidad de Oviedo, 33006 Oviedo, Spain
}

(Received 26 October 2004; in final form 2 December 2004)

\begin{abstract}
In this work we succintly review the main features of bone formation in vertebrates. Out of the many aspects of this exceedingly complex process, some particular stages are selected for which mathematical modelling appears as both feasible and desirable. In this way, a number of open questions are formulated whose study seems to require interaction among mathematical analysis and biological experimentation.
\end{abstract}

Keywords: Bone formation; Mathematical modelling; Human skeleton; Skeletal morphogenesis

\section{Introduction}

The human skeleton is a complex organ, composed of more than 200 different elements of various shapes, sizes and origins, that provides a physical framework for the body and carries out various important functions. For instance, it transmits the force of muscular contraction during movement, protects a number of internal organs and serves as a reservoir for ions, particularly calcium.

Skeletal elements are formed by two different tissues, cartilage and bone. Both have supportive functions, but are otherwise very different. The distinctive properties of cartilage include low metabolic rate, avascularity, capability for continued growth and high tensile strength coupled with resilience and elasticity. By contrast, bone constantly renews itself to meet both mechanical and metabolic demands, is highly vascularized and displays rigidity and hardness owing to the inorganic salts deposited in its extracellular matrix (ECM).

Skeletal morphogenesis displays a rich structure of coordinated events, which involve sophisticated signaling pathways. Its starting point, embryonic development, has been a key scientific subject since ancient times, and flourished during the nineteenth and twentieth centuries (see for instance Wolpert 1998 for a review [1]). Moreover, it has played a crucial role in the increasing interaction between Mathematics and Life Sciences that has taken place since the second half of the twentieth century. A milestone in this interplay is widely credited to Turing's seminal work [2] on mathematical modelling of morphogens (i.e. chemical substances whose diffusion and interaction are instrumental in producing spatio-temporal patterns). An overview of the current biological concepts on embryonic development can be found, for instance, in Wolpert [1]. We refer to that book for a detailed description of a number of technical terms that are mentioned in this article, as for instance the setting up of body axes, the origin and specification of germ layers: Ectoderm, mesoderm (and some particular blocks of that tissue known as somites), and endoderm. Particularly relevant are the concepts of cell specification, regulation and determination, which refer to the fact that the structure and performance of different cells are made precise at different time stages, some of which are susceptible to modification, while at the determination stage the cell fate is irreversible.

A key feature of the skeleton is that the nature of skeletal elements greatly varies among different locations of the body. Actually, the sizes and forms of bones need to be carefully shaped according to local requirements and mechanical loads. Skeletal formation is subject to regulation by a number of factors, which may be of a genetic, endocrine (induced by long-distance diffusibles) or paracrine (induced by short-distance ones) nature. Three main sequential processes can be distinguished during skeleton development. A first step consists in the differentiation of embryonic lineages that give rise to the overall pattern of the skeleton, as well as to the archetypal shape and identity of the individual elements. Second, once patterning has been established, the specific

*Corresponding author. E-mail: Miguel_Herrero@mat.ucm.es

ๆE-mail: jmlopez@uniovi.es

Journal of Theoretical Medicine

ISSN 1027-3662 print/ISSN 1607-8578 online @ 2005 Taylor \& Francis Group Ltd

http://www.tandf.co.uk/journals

DOI: $10.1080 / 10273660412331336883$ 
shape of a skeletal element is further defined in each location by endocrine or paracrine factors. Finally, when bone primordia with specific shapes have been formed, an increase in size occurs during a suitable growth period, which results in bones achieving their mature forms.

The goal of this work consists in describing the general process of bone formation, paying attention to its quantitative modelling (or, as will be often the case, the want of it). More precisely, a number of relevant steps will be highlighted, for which the need of mathematical models is stressed, as a way to identify and control the main players in each considered situation. Mathematical modelling not only provides a mean to compute quantitative features such as time and space scales, growth rates, etc. but moreover it helps to clarify which factor is required for each observed phenomenon. In this way, it may thus lead to a better understanding of the molecular processes involved by underlining, for instance, the need of ascertaining additional agents in the signalling pathways [3], or by identifying the way in which robustness against malfunctions arise [4].

While mechanical forces are crucial for the actual development and subsequent performance of bone elements, to keep this work within reasonable bounds we have decided to focus herein in the molecular agents involved, and the corresponding reaction-diffusion (RD) mechanisms.

We conclude this Introduction by describing the plan of this work. In section 2 we shall briefly recall some aspects of the early stage of skeletal formation. After sketching various relevant aspects of embryo development, we remark therein on the mathematical modelling of some of them: The formation of elongated organizers, the interaction among different regions of that type, and the onset of size-regulated condensates which eventually will develop into fully blown bones. We then turn our attention in section 3 to the transition from cartilage to bone in the so-called endochondral ossification process, that accounts for the formation of most of the bone pieces in vertebrates. Section 3 is mainly concerned with primary ossification, whereby bone tissue spreads radially from the centre of the bone template. The subject of secondary ossification (SO), which eventually leads to bone formation at the bone ends, is then considered in section 4 .

Among the many interesting features of these processes, is that a key role is played by angiogenesis (the recruitment of blood vessels from a preexisting vasculature). Indeed, transformation of cartilage into bone requires changing the avascular nature of the cartilaginous tissue into a highly-vascularized bone, a fact which is mediated by the action of a number of angiogenic factors. As will be recalled below, the process of bone formation bears deep analogies with tumour invasion, which only adds further interest to understanding the way in which bone growth is triggered and regulated under physiological conditions. The reader is referred to Carmeliet [5], Yancopoulos et. al. [6] and Bussolino et. al. [7] for descriptions of angiogenesis on both homeostatic and malignant situations. Mathematical models of angiogenesis have been extensively studied in recent years. We refer to Sleeman and Levine [8], Levine and Sleeman [9], Chaplain and Anderson [10] and references therein for details on this last topic. Also, when dealing with the later stages of bone formation, a useful tool to describe bone invasion is the concept of travelling wave, which naturally leads to the question of characterizing the RD systems that can support them. Some of these are briefly discussed below, and a number of open questions (concerning both the biological and mathematical modelling of such processes) will be stated. Finally, we summarize our approach, as well as some of the questions raised, in a short section 5 at the end of the paper.

\section{Skeletal morphogenesis}

The skeleton emerges from cells of three distinct lineages. In fact, the craniofacial skeleton is formed by cranial neural crest cells, the axial skeleton is derived from paraxial mesoderm (somites) and the limb skeleton is the product of lateral plate mesodermal cells. These cells proliferate and concur to establish over time the three coordinate axes along which the skeleton unfolds: Proximal-distal (PD), antero-posterior (AP) and dorsalventral (DV).

A key open question is to ascertain the way in which cells derive positional information. It has been suggested that this occurs progressively in a PD sequence [11]. Alternatively, information regarding the number and anatomic identity of skeletal elements might have been already specified in the corresponding cell lineages by the time of their appearance [12]. In any case, the family of transcription factors encoded by homeobox genes seems to play a key role at this early stage of development.

\subsection{Models of pattern formation}

A large part of the experimental work conducted so far in skeletal formation has been concerned with limb development in chicks. In this context, the formation of the limb involves the onset of differences along the three main axes: AP (from the thumb to the little finger), DV (from the back of the hand to the palm), and PD (from shoulder to hand). Experiments made on removing and transplanting cell regions have established that the AP axis is determined first, followed by the DV one, whereas the PD axis is determined at the last stage. This stereotyped patterning sequence is well conserved among tetrapods [13].

In the case under consideration, the first evidence of limb formation consists of the proliferation of somatic mesoderm cells along the length of the embryo. These cells progressively accumulate under the ectoderm to form a longitudinal region known as the Wolffian ridge. Proliferation is higher in the pectoral and pelvic regions, and this results in the formation of limb buds. At this 
stage, the limb tip is covered with a sheet of thickened ectoderm called the apical ectodermal ridge (AER) (see plate 1 in figure 1), and the limb tip elongates by continuous proliferation of undifferentiated mesenchyma. The region consisting of rapidly dividing cells adjacent to the AER is called the progress zone (PZ).

How can a thin and relatively long region as the AER be generated? A model due to Meinhardt [14,15] proposes that a thin organizer can be generated at the boundary between two regions with different cell determinations, each of which has been established at a preceding step. Along such a boundary, morphogens would be produced

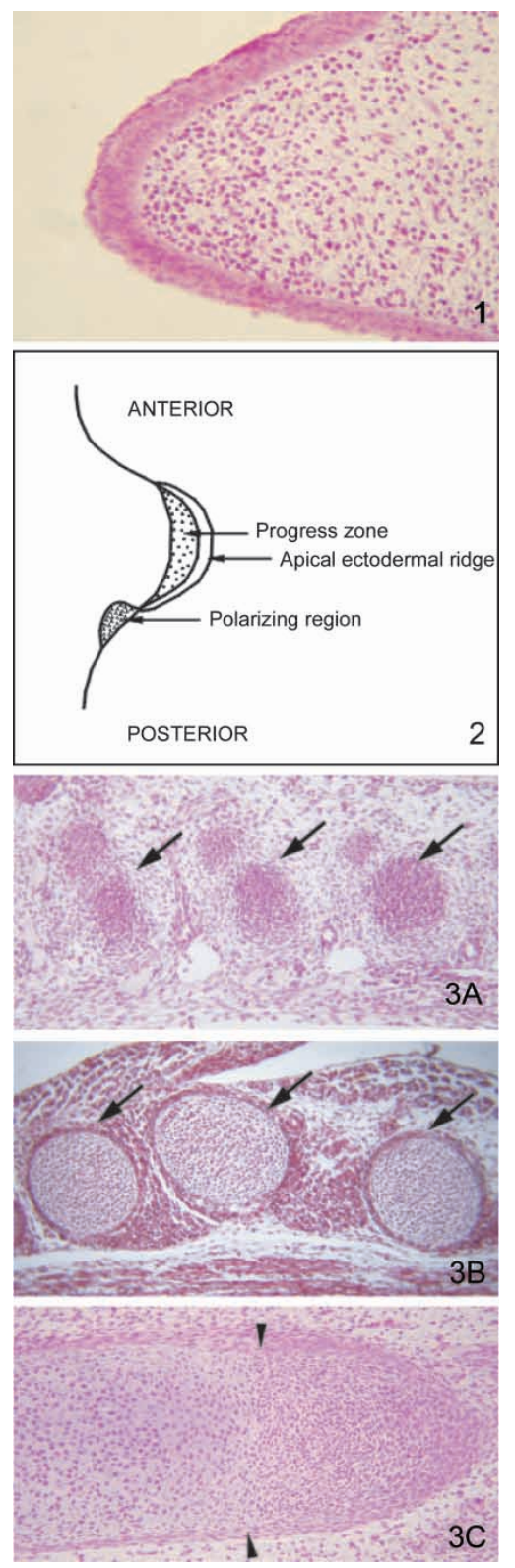

Figure 1. Aspects of bone morphogenesis. 1: Embryo limb bud sectioned along the proximodistal axis. 2: A sketch of the organizing zones in the limb bud. 3: Progressive stages of development of cartilage elements. Condensation of mesenchymal cells (3A) differentiate into chondrocytes to produce bone primordia formed by cartilage (3B). A key feature of mesenchymal differentiation into chondrocytes is the production of extracellular matrix (3C, arrowheads). as a result of cooperative interaction among cell types located in the adjacent regions. In particular, the local concentration of morphogen(s) provides a measure for the distance of a cell from such a boundary, and is therefore a convenient way to yield positional information. As described in detail in Meinhardt [15], and checked experimentally by Maden [16], this model is able to account for a number of otherwise puzzling experiments on tissue grafting.

Limbs have to be inserted at particular positions along the AP and DV axes. The generation of positional information for the DV patterning requires a longextended midline as a reference line for that purpose. It is not obvious how an organizing region with a long extension along the AP axis, and a short perpendicular extension, should emerge. An analysis has shown that this requires the collaboration of two pattern-forming systems [17]. In vertebrates, for instance, a moving organizer (e.g. Hensen's node) leaves behind a stripe-like structure, the notochord. At the mathematical level, the building block of such model is the concept of activator-inhibitor system [18]. In its simplest setting, it can be described by two coupled differential equations of the form:

$$
\begin{gathered}
\frac{\partial a}{\partial t}=D_{a} \Delta a+\rho_{a}\left(\frac{a^{2}}{h}-a\right) \\
\frac{\partial h}{\partial t}=D_{h} \Delta h+\rho_{h}\left(a^{2}-h\right)
\end{gathered}
$$

Here $a=a(x, t)$ stands for the concentration of an activator, an autocatalytic substance that also enhances the production of an antagonist $h=h(x, t)$ (the inhibitor). Autocatalysis (or self-enhancement) is needed for small local inhomogeneities to be amplified, but does not suffice to produce stable patterns since such property, if unchecked, would lead to overall activation. To localize the pattern, lateral inhibition has to be introduced in the model, and this can be achieved by requiring that $D_{h} \gg D_{a}$ in the system above. It has been shown that activatorinhibitor systems can produce a number of geometrical patterns, including stripes, spots and tesselations [19].

Following Meinhardt [17], a line of activation can be obtained by coupling a stripe-generation system as for instance:

$$
\begin{gathered}
\frac{\partial a}{\partial t}=\frac{s\left(a^{2}+b_{a} c\right)}{b\left(1+s_{a} a^{2}\right)}-r_{a} a+D_{a} \Delta a \\
\frac{\partial b}{\partial t}=s\left(a^{2}+b_{a} c\right)-r_{b} b+D_{b} \Delta b+b_{b}
\end{gathered}
$$

with a spot-generating system given by:

$$
\begin{gathered}
\frac{\partial c}{\partial t}=\frac{R_{c}\left(e c^{2}+b_{c} a\right)}{d}-r_{c} c+D_{c} \Delta c \\
\frac{\partial d}{\partial t}=r_{d} e c^{2}-r_{d} d+D_{d} \Delta d+b_{d}
\end{gathered}
$$


where $e$ is a substance removed by the stripe system:

$$
\frac{\partial e}{\partial t}=b_{e}-r_{e} e-s_{e} a e+D_{e} \Delta e
$$

Roughly speaking, for a suitable window of values of the parameters appearing in the model, the $(a, b)$ system generates stripes due to the saturation of the autocatalysis by means of the term $s_{a}>0$. Due to a baseline inhibitor production (represented by the term $b_{b}$ ), growth in $a$ requires triggering from the spot system (produced by the term $b_{a} c$ ). The second set of equations generate spots due to the absence of saturation $\left(s_{a}=0\right.$ in the equation for $c$ ). The maximum of $c$ becomes regularly displaced as a consequence of the fact that autoregulatory formation of $c$ depends on a substrate $e$, that is removed by the stripe system. The local destabilization of the spot maximum thus leads to its motion in front of the tip of an incipiently formed straight line. Note that curvature effects are not included in this model.

Early during the elongation, the more proximal (i.e. the innermost) of these mesenchymal cells show diminished mitotic activity, and begin to condensate in the core of the limb to form the cartilaginous elements that will act as a template for the skeleton. Skeletal elements differentiate in a PD direction: The first cartilage to appear in the upper limb is the humerus, followed by the radius and ulna, and then by the wrist elements and the digits. Concomitantly, muscle cell precursors condensate peripheral to the cartilage units to form the muscle masses.

The AER is crucial for the continued outgrowth of the limb, since this structure induces cell proliferation of the subjacent apical mesoderm. Actually, if the AER is removed, the underlying mesoderm ceases to divide, and the limb outgrowth is arrested. On the other hand, an additional limb element emerges when a supernumerary AER is grafted to the side of the young limb. Furthermore, if an AER is combined with limb mesoderm in tissue culture, the mesoderm is induced to proliferate. The relationship between AER and mesoderm is a two-sided one. Indeed, if limb mesoderm is removed from an early limb, leaving an ectodermal jacket that is then packed with non-limb mesoderm, the AER regresses, and the mesoderm stops proliferating. Conversely, an AER forms, and a supernumerary limb develops, when prospective limb mesoderm is grafted beneath flank ectoderm. This happens according to a clear temporal pattern. In the wing of the chick, the limb mesoderm develops this inductive capacity by the so-called Hamburger-Hamilton stage 13 (about 40 to $45 \mathrm{~h}$ after fecundation, a.f.), and loses it by stage 31 (day 3 a.f.). On its turn the leg mesoderm follows a similar schedule slightly later. These findings suggest that the AER emits a signal that stimulates cell division in the subjacent mesenchyma, whereas the mesoderm is thought to produce a factor that is responsible for the presence of the AER, although the corresponding signaling pathways have not been elucidated as yet. It is known, however, that the AER can be induced and preserved by members of the fibroblastic growth factor (FGF) family [20].

\subsection{Establishment of the developmental axes}

The limb is an integrated structure, and its development requires the cooperative unfolding of its components along its three main axes. However, the peculiarities of their respective formation are best captured by examining each case separately.

2.2.1 PD axis. After the induction of the AER, further limb development depends on signals emanating from that zone. As a matter of fact, experiments by Rowe and Fallon [21] have shown that if the AER is removed at a successively later stage, progressively more distal elements will appear in the truncated limb. More precisely, when the AER was removed from a wing bud at day 3 a.f., only a humerus was formed in the stump. However, when the AER was amputated a day later, the radius and ulna also differentiated in the resulting wing. These facts imply that the cartilaginous elements in the limb are laid down in a PD direction. Experiments of removing AER and transplanting it to a younger limb bud did not produce more distal elements, but a young bud gave raise to the full complement of limb elements regardless of the age of the AER grafted to it. Thus, the AER is important in maintaining mitotic activity in the apical tip mesoderm and in sustaining continued outgrowth, but does not suffice to determine the positional properties of the mesoderm.

At this juncture, it is worth observing that, as recalled by de Lise et al. [20], implantation of beads soaked in FGFs can substitute for the AER and maintain PD growth. This suggests that the FGFs secreted from the AER may be responsible for keeping the PZ active and the subsequent limb outgrowth, a process during which the thickness of the PZ zone remains constant. The cells that escape the PZ first differentiate into the most proximal limb elements, while cells that remain longer within the PZ will become distal elements. Experiments in which the tip of a very young limb was removed, leaving behind a stump previously specified as humerus, and was replaced by a tip from a older limb that should have had only the more distal positional values (wrist and digits) in its PZ, resulted in a wing with the intermediate values (radius and ulna) missing. Alternatively, when the tip from a young limb (which should contain all or nearly all of the positional values in its PZ) was grafted onto an old stump (which should have most of the limb values, except the most distal ones, fixed), a composite limb resulted in which all the intermediate values were duplicated. These experimental results have two important implications:

(1) Once mesoderm cells have left the PZ, their fate is irreversibly fixed,

(2) The positional information obtained by a cell is determined by the time spent at the PZ, under control from the AER, as proposed earlier by Summerbell [22,23]. 
The way in which the PZ might confer positional information to the mesoderm cells seems not to be completely understood. Experiments of irradiation with $\mathrm{X}$-rays have reported that the number of rounds of cell division that a cell undergoes in the PZ determines whether it will become a proximal or a distal element [24]. Nevertheless, when using landmarks to track the fate of particular parts in a developing organ that allow to distinguish host and graft tissues, it was found that positional values were not always irreversible, and some regulation occurred. In this way, stump tissue might be respecified to form more distal or proximal values [25]. Such disparity of results may be due, at least in part, to the ages at which the grafts are implanted. In particular, regulation can occur if the deletion experiments are done on young embryos (less than three to four days in the case of chick embryos), whereas positional values become fixed when the operations are performed in older limbs (more than four days in the previous case, see Mercader et al. [26]).

We point out that an alternative approach accounting for the formation of the PZ zone, as well as for the presence (or absence) of proximodistal intercalation after certain graft experiments in amphibian limbs, has been proposed by Meinhardt [27]. In this model, a morphogen responsible for the PD axis is produced at the AER. This substance would induce the structures in the underlying mesodermal cells, in such a way that higher concentrations give raise to more distal determinations. On their turn, mesodermal cells would produce an apical ectodermal maintenance factor (AEMF), a diffusible product, which controls the PD-morphogen production of the AER. The model assumes that more distally determined cells produce more AEMF. Distal determination is considered to be irreversible; however, whenever the morphogen concentration becomes higher than required for the original cell determination, a new element, in the PD-sequence is formed.

2.2.2 AP axis. The mechanisms involved in the formation of the AP axis are different from those recalled in our former paragraph. Experiments showed that when a small piece of tissue is excised from the most posterior part of the limb at the junction with the body wall (the so-called zone of polarizing activity, ZPA), and then grafted into the anterior margin of another limb, this resulted in duplication of digits in the limb receiving the graft. Furthermore, these duplicated digits were arranged in mirror symmetry with respect to the host digits (cf. [1]). The ZPA is presumed to be the source of one (or several) morphogen(s) which would diffuse across the limb to form a concentration gradient that might determine the AP axis.

The first clues as to the nature of such morphogens came from a study by Maden and Mustafa [28], who found that if amputated salamander limbs were bathed in retinoic acid (RA), the limbs regenerated, and had duplicated limb parts (e.g. two sets of radius and ulna growing end-to-end).
Subsequently, Tickle et al. [29] and Summerbell [30] showed that RA could bring about the same duplications of digits as could be produced by an additional ZPA. Later, Thaller and Eichele [31] demonstrated that RA was indeed present in the chick limb, displaying a gradient with highest values located at the posterior margin. Recently, Mic et al. [32] have reported the existence of two phases of RA signalling required for vertebrate limb development. Their study shows that limb RA synthesis is under control of retinaldehyde dehydrogenase-2 (Raldh 2) expressed in the lateral plate mesoderm, which generates a proximodistal RA signal during limb growth.

Finally, we remark that, as recalled by Wolpert [1] other molecules may be instrumental in the signaling process just mentioned. For instance, the sonic hedgehog protein (Shh), which is known to be involved in several patterning processes, is expressed at the ZPA, and growth factors belonging to the BMP (bone morphogenetic protein) family have been shown to be present in a gradient above the PZ, with largest values located at the posterior margin of the limb bud. Numerical simulations of growth in two space dimensions (corresponding to the $\mathrm{AP}$ and PD axes) have been performed by Dillon and Othmer [33] (see also Gadgil, Dillon and Othmer [34]). These authors consider a simplified setting in which only two diffusible morphogens are considered, which are, respectively, produced at the AER and the ZPA regions. The limb bud tissue is taken to be a viscous fluid, and cell growth and division are introduced by means of a distributed source of volume (depending on the morphogen concentrations) within the limb bud. The moving limb bud boundary is postulated to move with the local fluid velocity, and the boundary forces are transmitted directly to the fluid by a force density located at the boundary. As pointed out by the authors, their model allows for introducing additional elements, and can be used to test signal transduction pathways for morphogen signalling. A plot of the relative positions of the AER, PZ and ZPA is provided in figure 1, plate 2.

2.2.3 DV axis. This situation has received relatively little experimental attention compared with the previous two cases. From the scarce data gathered, it appears that this axis is specified by cells derived from both the mesoderm and the ectoderm, although at different stages of development. When fragments of limb bud mesoderm are dissociated and centrifugally compacted limb bud cells are repacked into the ectodermal hull of a three to four day wing bud, and are then grafted to the flank of a host embryo, the skeleton and musculature of the distal elements have a DV axis conforming to that of the ectoderm. Similarly, if intact mesodermal cores of chick embryo leg buds of the same age are recombined with the ectodermal hulls, so that the DV axis of the ectoderm is reversed, the musculature and skeleton will also be reversed along the DV axis. The ectoderm can specify the DV axis as early as 50 to $53 \mathrm{~h}$ a.f., before the AER has even 


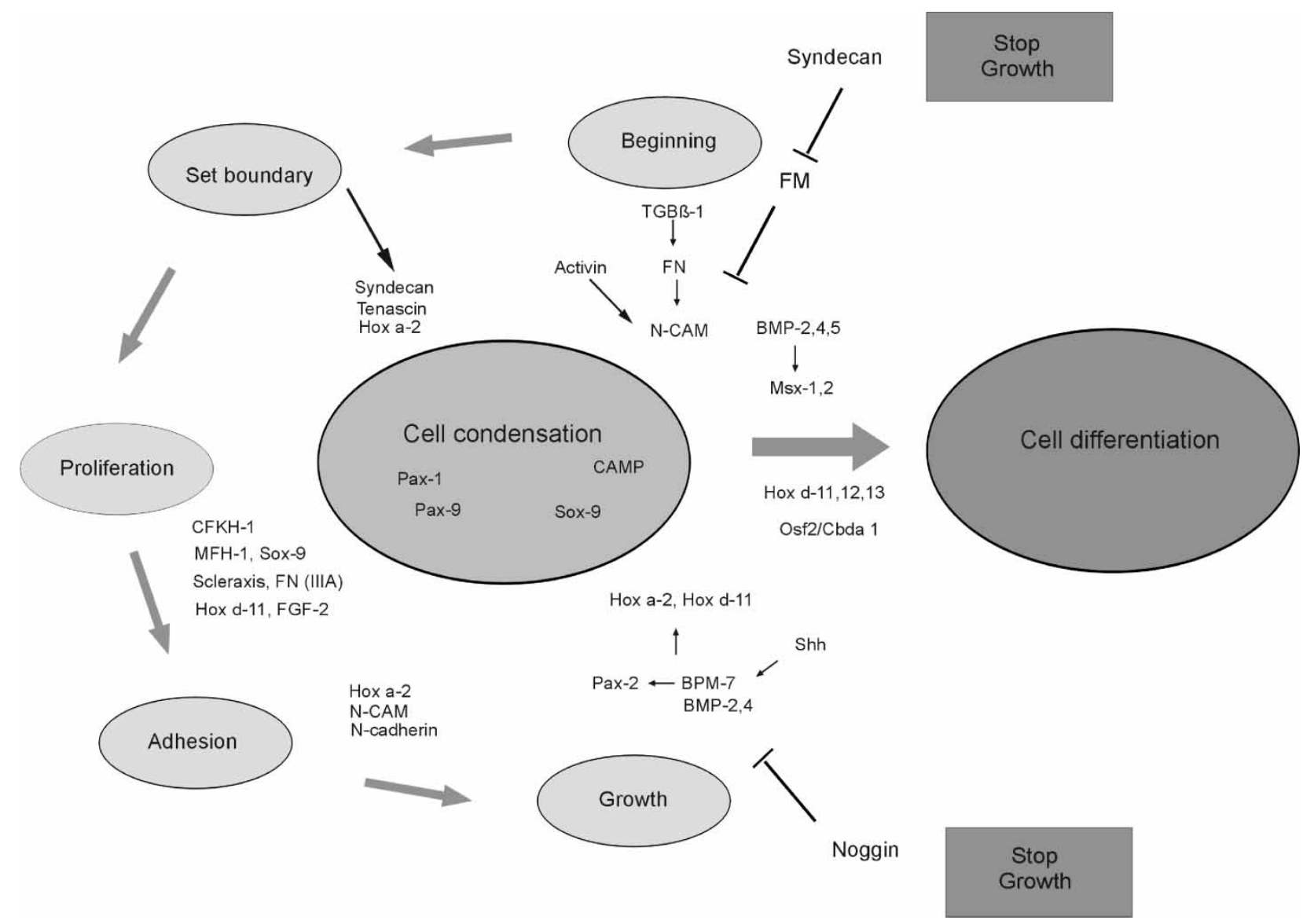

Figure 2. A sketch of the processes leading to cell condensation.

appeared. See Wolpert [1] and de Lise et al. [20] for a review of genes involved in DV patterning.

\subsection{Cell condensation}

Once skeletal cell lineages are defined, they migrate to locations in the embryo where they proliferate and aggregate to form cell condensations prefiguring the outlines of the various skeletal elements. Such condensations develop in PD sequence and the cells, while still undifferentiated, are more closely packed in the core than in the peripheral areas. The mechanisms of cell condensation have been reviewed by Hall and Miyake [35] (see also [20,36]), who have stressed the following stages of the overall process.

(A) Positional information seems to provide a pattern for the distribution of condensation centres, toward which cells aggregate (or fail to disperse from).

(B) Boundaries of condensation are established, and the corresponding borders are characterized as regions where some substances are expressed. In particular, separation between contiguous condensation centres may be regulated by diffusible signals released by the cells themselves; see 3 in figure 1.

(C) A critical condensation size is required to decide whether skeletogenesis will be initiated. In other words, a size threshold seems to exist, below which further development is not viable, and above which abnormally large skeletal elements can form. A question that then arises is that of deducing the size of an aggregate from the knowledge of the signalling process leading to its formation.

As far as we know, there is only one biologicallymotivated system for which an estimate on the size of condensates has been derived from first principles. This is the case of the so-called Keller-Segel system (KS) [37], which was proposed as a model to account for aggregation in the slime mold Dictyostelium discoideum (Dd). This reads as follows:

$$
\begin{aligned}
& \frac{\partial u}{\partial t}=D_{1} \nabla^{2} u-\theta \nabla(u \nabla v) \\
& \frac{\partial v}{\partial t}=D_{2} \nabla^{2} v+A u-B v .
\end{aligned}
$$

In this continuum model, $u(x, t)$ and $v(x, t)$ denote, respectively, the concentration of Dd cells and that of a chemical (cAMP: cyclic adenosine monophosphate) that is released by the cells and induces aggregation. As a matter of fact, Dd cells are known to emit pulses of 


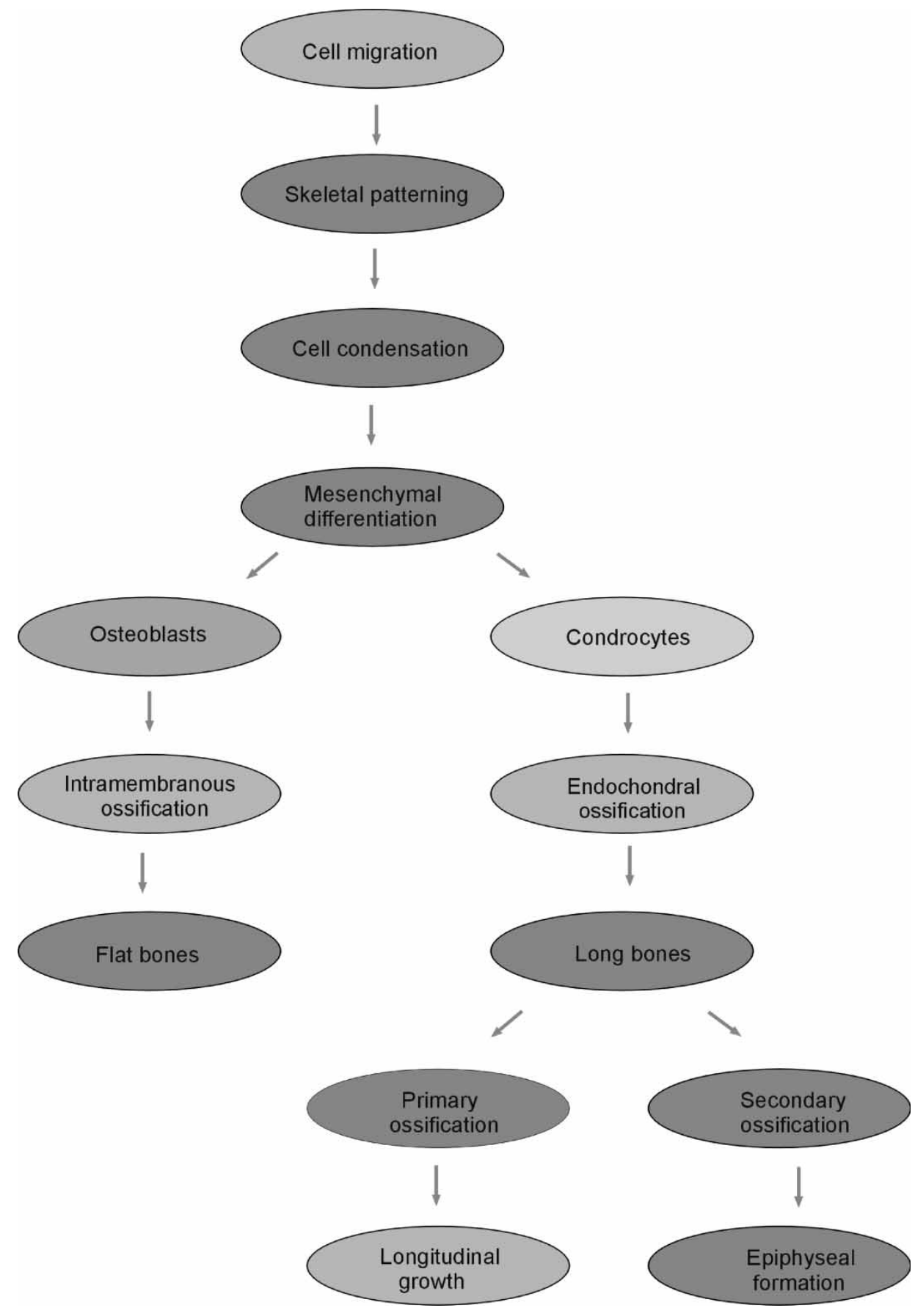

Figure 3. An overview of the overall process of bone formation.

cAMP when food becomes scarce, to subsequently move upwards gradients of such substance, heading towards condensation centres where they gather in rather fixed quantities (see [38,39], cf. also [40,41] for reviews on mathematical properties of KS). In the former equations, $D_{1}$ and $D_{2}$ are the respective diffusion coefficients, $\theta$ is a positive motility parameter, and $A$ and $B$ are production and decay parameters, respectively. Despite their apparent simplicity, these equations possess a size-regulation property: as soon as a minimal threshold density is reached, cells gather together in aggregates with a precise size, irrespective of how large the initial population is. More precisely, in two space dimensions such a system has a positive solution for which the cell density $u(x, t)$ concentrates around a single point $x_{0}$ in the form of a Dirac delta, $M \delta\left(x-x_{0}\right)$ having a mass $M$ given by:

$$
M=\frac{8 \pi D_{1} D_{2}}{A \theta}
$$

(see Herrero and Velázquez [42]). Furthermore, the corresponding size-regulatory mechanism is stable under 


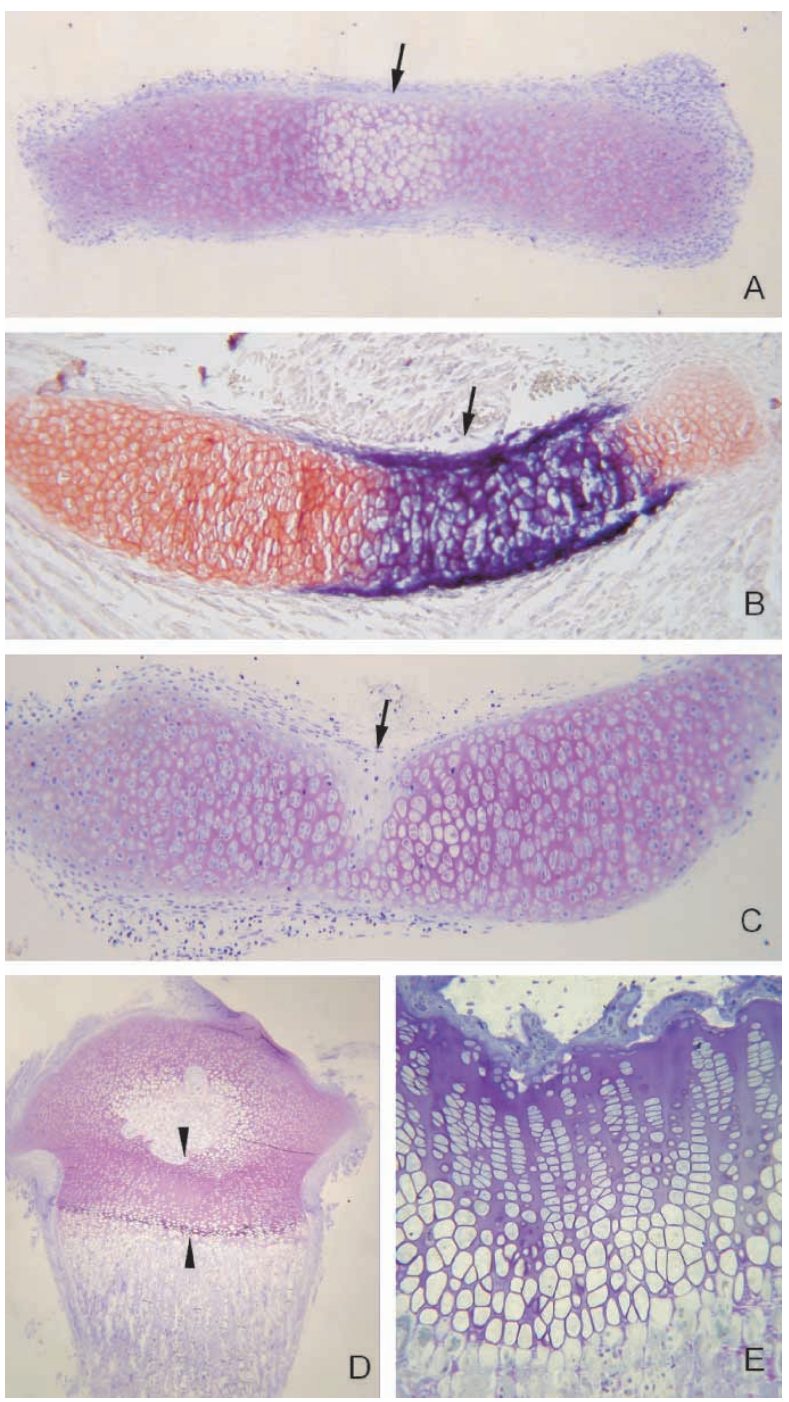

Figure 4. Progressive stages in the ossification of a long bone. Cartilage hypertrophy begins at the center of the template (A, arrow) where extracellular matrix begins to calcify (B, blue stain). Vascular mesenchyme enters the cartilage and divides the template into two zones of ossification (C). Blood vessels and mesenchyme enter the epiphyseal cartilage to form the secondary ossification center (D). Primary and secondary centers are separated by a layer of cartilage, the growth plate (D, arrowheads). The growth plate is typically organized into vertical cell columns (E).

perturbations [43]. It is natural to wonder whether a similar result could be obtained for the condensation process under consideration here. Checking this point might require a detailed analysis of mathematical models as those recently derived by Hentschel et al. [44] to describe precartilage condensation.

(D) Within any condensate, cells have to adhere to each other. Moreover, a precise timing is required for its formation, and a particular shape is eventually achieved.

A summary of genes and gene products involved in this process, as well as a scheme of their interaction, is provided in figure 2 which is adapted from a similar one by Hall and Miyake [35].
We finally point out that condensation occurs close to pre-existing blood vessels. In such aggregates, both proliferation and apoptosis (programmed cell death) are particularly active, the first one taking place mainly at the border of the condensate. Aggregation is eventually followed by differentiation, whereby mesenchymal cells become osteoblasts (osseous cells) or chondrocytes (cartilaginous cells). When cells differentiate directly into bone-forming osteoblasts, the process is termed as intramembranous ossification: this is the way bones of the craniofacial skeleton are generated. However, most mesenchymal condensations differentiate into chondrocytes, and then yield a framework of cartilage templates that produce a transient skeleton. The overall process is sketched in figure 3, also adapted from Hall and Miyake [35].

\section{Bone growth}

As we have recalled at the end of the previous section, intramembranous bones grow by peripheral addition of new osseous tissue at the osteogenic fronts. This is a relatively simple process, which only allows for low rates of bone growth. By contrast, endochondral ossification is a more complex phenomenon that permits high rates of longitudinal bone growth. Indeed, the growth of endochondral bones is the result of two tightly coupled processes: continued and vectorial production of cartilage tissue, and replacement of a part of it by osseous tissue. As a consequence, a strict coordination of cartilage enlargement, cartilage resorption and osseous tissue formation is required. We shall briefly recall the main stages in endochondral bone growth in the sequel. For more detail, the reader is referred to Hunziker [45], Rivas and Shapiro [36] and Kronenberg [46].

\subsection{The starting of endochondral ossification}

The beginning of the process is characterized by the appearance of differentiated chondrocytes in the central portion of the mesenchymal condensations. Cells within these regions begin to secrete, and become surrounded by, a specific ECM containing type-II collagen, whereas the developing joints between each cartilage template (i.e. every condensate which eventually will become a bone) show a persisting cellular interzone region containing a homogeneous aggregation of undifferentiated cells, as depicted in figure $1,3 \mathrm{C}$.

Shortly after cartilage differentiation occurs, chondrocytes in the centre region of the template start to grow larger in size (thus becoming hypertrophic chondrocytes, $\mathrm{HC}$ ), and to secrete and organize a different type of ECM. A layer of undifferentiated cells that differentiate into osteoblasts surrounds HC. These last begin to secrete osseous matrix, and produce intramembranous, periosteal bone formation at the periphery of the equator of the cartilage template. As a next step, periosteal buds 

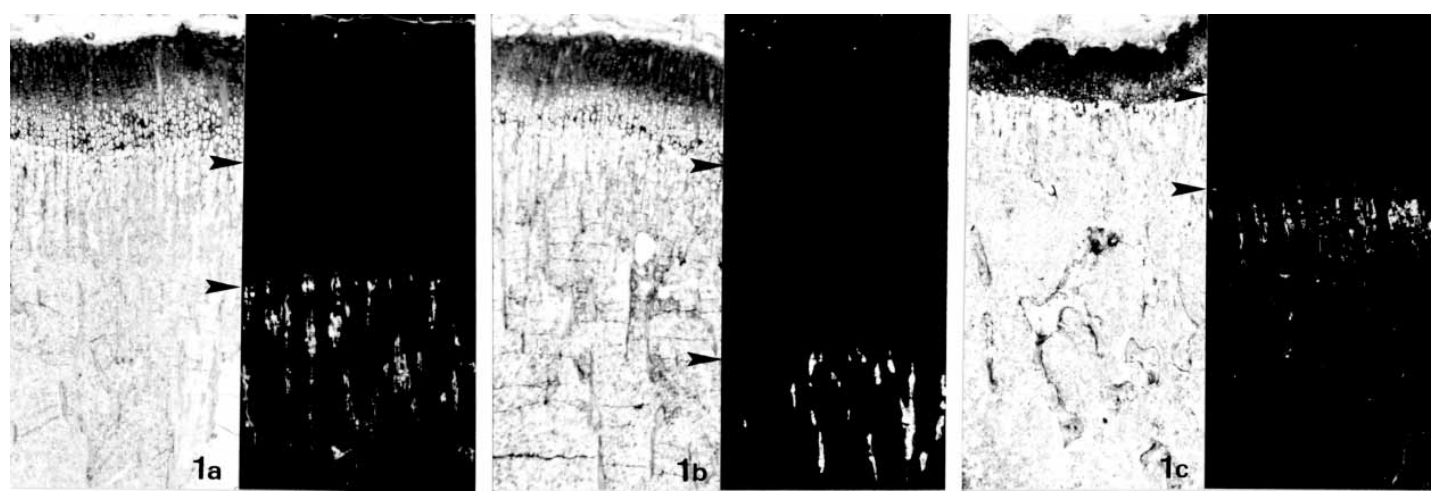

Figure 5. Sections of the proximal tibial growth plate viewed by bright-field microscopy (left) and by incident-light fluorescence microscopy (right) of 21- (A), 35- (B), and 80-day-old (C) rats injected with calceine four days before sacrifice. Calceine is a fluorescent label that binds specifically to mineralized matrix at the time of application and the distance between the zone of vascular invasion in the growth plate and the proximal end point of the calceine front (arrowheads) indicates longitudinal bone growth. Growth rates show the highest values in 35-day-old rats, the lowest values in 80-day-old rats, and intermediate values in 21-day-old rats. Reproduced from J. Bone Miner. Res. 2000;15:82-94 with permission of the American Society for Bone and Mineral Research.

containing blood vessels and perivascular mesenchymal cells invade the middle of the template. This angiogenic process signals the replacement of cartilage by bone, and gives raise to the formation of the primary ossification centre (POC).

In addition to this central invasion, cartilaginous templates are subsequently penetrated at their lateral ends (epiphyses) by invading blood vessels and osteogenic cells. In this way, secondary ossification centres (SOC) are formed at each distal extreme of the template. POC and SOCs remain separated during the period of active bone growth by a thin layer of cartilage, the so-called metaphyseal growth plate. Activity at the POC results in longitudinal bone growth, whereas that of the secondary centres leads to the formation of two roughly spherical epiphyses at the ends of the template. These are crucial to form movable joints between adjacent bones. The stages shortly summarized above are illustrated in figure 4 .

\subsection{Primary ossification}

This is the first step in the transformation of a cartilaginous template into a mature osseous skeletal element. We have already recalled how a POC is formed. Within this centre, ECM is degraded, hypertrophic chondrocytes undergo apoptosis, osteoblasts replace the disappearing cartilage by trabecular (i.e. grid-like structure) bone, and bone marrow is formed. At the same time, osteoblasts in the perichondrium form a collar of compact bone around the middle portion (diaphysis) of the template, so that the POC ends up being located inside a tube of bone.

As replacement of cartilage by bone spreads in both directions, two invasion fronts between the newly formed bone and the remaining cartilage are established. Activity at the POC yields a continuous production of new osseous tissue that invades cartilage. This last does not disappear, however, since the process of cartilage resorption is balanced by a process of continued cartilage production. As a result, the amount of cartilage remains fairly constant at this stage, whereas an increase in the osseous tissue, resulting in bone growth, is observed. This invasive and destructive process is very much reminiscent of malignant tumour growth, except that it operates on a physiological and controlled basis, which is finely tuned to ensure suitable tissue production.

Cartilage generation is determined by a combination of chondrocyte proliferation, ECM synthesis, and cellcontrolled phenotype modulation (hypertrophy), and is subject to complex regulation by endocrine and paracrine factors. A gradient of differentiating chondrocytes is formed from the periphery to the centre of the template. The layer more distal from the resorption front is called the reserve cell zone (sometimes also termed as the stem, germinal or resting cell zone). It contains spherical cells with little or no cell division. The reserve zone impinges on the proliferative zone, where cells rapidly divide and give rise to columns of flattened cells secreting hyaline ECM rich in type-II collagen. After having run through a number of cell divisions, proliferating chondrocytes lose their capacity to divide, and begin hypertrophy. The area where this occurs is named the hyperthrophic zone. Hypertrophy contributes efficiently and economically to longitudinal bone growth at the cellular level. In this way, the layer more proximal to the resorption front contains hyperthrophic chondrocites having a calcified ECM, and is particularly suited for vascular invasion.

As long as cartilage keeps on growing, the bone template increases in length. At the time sexual maturity is reached, high levels of estrogen and testosterone leads all chondrocytes to hypertrophy. Then all cartilage is replaced by osseous tissue, and bone growth definitely ceases.

Concerning time scales, several stages can be distinguished. First, the central zone of the model is invaded by blood vessels and osteoprogenitor cells, to eventually produce a small cylinder of bone tissue at the template centre. This happens relatively fast (in about $48 \mathrm{~h}$ in the rat tibia). A second stage, termed as the stationary period, is characterized by one-directional 
expansion of bone from the model centre to each side. During this period (which is the longest one, lasting about 4 to 5 weeks in the rat tibia), bone and cartilage size are sharply increased, the first being larger than the second, so that the size of the cartilaginous zone diminishes. A final stage corresponds to a slowing down of both velocities, so that the growth process is eventually brought to a stop [47]. These facts are recalled in figure 5.

The transition from cartilage to bone is achieved at the so-called growth plate, a zone of about 600 microns width in rat tibia, which displays a high anysotropy and a distinct cellular organization [45,48]. As depicted in the figure 4(E), its structure can be described as consisting of vertical fingers made up of chondrocytes piled up as a stack of coins, each surrounded by ECM. Every column shows the various stages a chondrocyte goes through during its life cycle, from hypertrophic specimens at the bottom to proliferating ones at the top of each stack.

\subsection{Modelling bone invasion}

At the local level, the bone invasion front is the playground of a number of chemical agents. The cartilage region is completely avascular, due to the presence of angiogenic inhibitors in that tissue [49]. In order for bone progression to occur, a fundamental change in the cartilage close to the growth plate has to take place: it has to switch from excluding angiogenesis to favouring it. This change is mediated by a phenotypic modification in chondrocytes at the cartilage region being approached by bone. Namely, chondrocytes not only become hypertrophic, but they also start to secrete a number of substances, which drastically alter the ECM around them. In particular, metallo-matrix proteases (MMPs) are released that corrode the ECM, thus facilitating the passage of blood vessels. Also, ECM features at this stage a component (type $\mathrm{X}$ collagen), which is unique to this process [50]. Furthermore, chondrocyte hypertrophy is accompanied by the release of angiogenic factors, as vascular-endothelium growth factor (VGEF), which attracts blood vessels. The ECM, a gel-like substance possessing a grid-like structure (at the electronic microscopy level) consisting of vertical and horizontal trabeculae, is then penetrated by blood vessels in its vertical direction, since horizontal trabeculae are easier to degrade. Invasion thus proceeds along a number of roughly parallel channels, as represented in figure 6 .

As a matter of fact, the process described contains some, but not all, of the main agents of the situation as is currently known. For instance, a number of angiogenic inhibitors of VGEF (as for instance, sexual hormones) are known to be turned on at appropriate moments of the animal life, with the effect of slowing down, and eventually arresting, bone growth.

The nature of the phenomena under consideration hints at a possible description by means of a mathematical model exhibiting travelling waves (TW). These are functions that move at a given speed without changing their shape (see for instance Mikhailov [51] and Herrero [40] for reviews on TW). As markers for a scenario of bone invasion triggered by the POC, a minimal model could include an angiogenic growth factor (VGEF), a MMP, and an angiogenic inhibitor. Let us denote these quantities (as well as their respective concentrations) by $u(x, t), w(x, t)$ and $v(x, t)$, respectively. Here $x$ represents a one-dimensional length variable, parallel to a given vertical trabecula of the ECM, and $t$ stands for time. We thus particularise our discussion to a given channel among two adjacent vertical trabeculae, and denote by

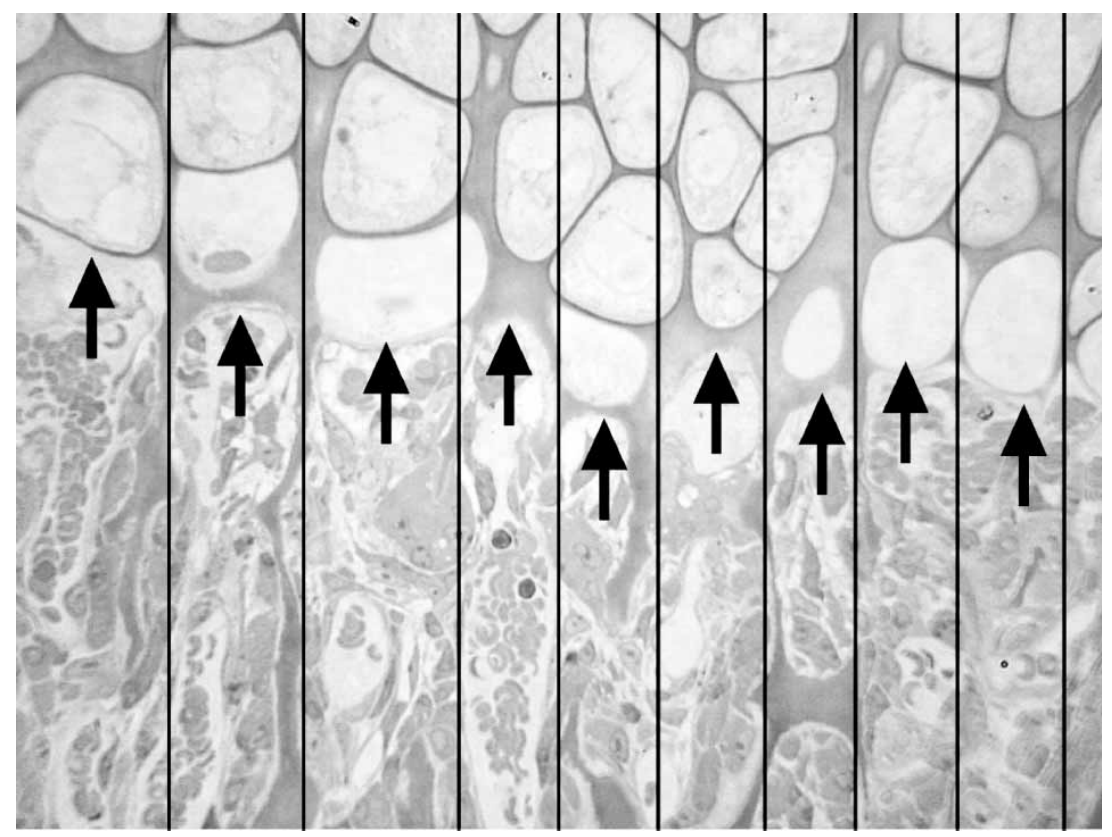

Figure 6. Micrograph of the lower hypertrophic zone and the vascular invasion front. There is an invading capilar sprout by each column of chondrocytes. 
$x=0$ the initial (proximal) position of the blood vessel located there: $x=L>0$ then corresponds to the final (distal) position of the blood vessel when the invasion stage under consideration is completed. In view of the data available (see for instance Alvarez et al. [47]), the gradients of $u, v$ and $w$ should be qualitatively similar to the graphs shown in figure 7.

When one looks for mathematical models that could account for such behaviours, a model scenario naturally unfolds. To begin with, waves as those expected for $u$ and $w$ are characteristic of the so-called excitable systems [51,52]. In its simplest setting, an excitable system can be thought of as consisting of a bistable system (possessing two stable equilibria separated by a third, unstable one) plus a restoring mechanism. Excitable systems admit solutions whose graphs are as those depicted in figure 7 for $u$ and $w$ : they can move in such a manner that one of the stable equilibria (say zero) is approached both ahead and behind the excited part of the function. These waves are usually termed as pulses. A typical example of excitable system is:

$$
\begin{aligned}
& \frac{\partial u}{\partial t}=D_{u} \frac{\partial^{2} u}{\partial x^{2}}+f(u)-w \\
& \frac{\partial w}{\partial t}=D_{w} \frac{\partial^{2} w}{\partial x^{2}}+a u-b w
\end{aligned}
$$

where $f(u)=u(k-u)(u-1)$, with $0<k<1$. However, the model to be envisaged should display additional features. For instance, the pulse for $w$ has to precede (and actually trigger) that of $u$, and both are expected to move with same speed. Moreover, a receding front (that is, a wave connecting a stableand invading - state to an unstable one) should describe the profile of the inhibitor concentration $v$. The simplest equation displaying this last type of behaviour is the Fisher-KPP equation 1 [53,54], which reads as follows:

$$
\frac{\partial v}{\partial t}=D_{v} \frac{\partial^{2} v}{\partial x^{2}}+v(1-v)
$$

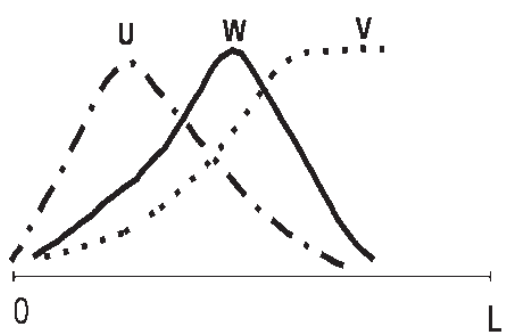

A
At this juncture, it is worth recalling that excitable systems have a very limited range of possible wave speeds (in many cases, just one), cf. Grindrod [55], and Mikhailov [51]. On the other hand, infinitely many wave speeds $c$ are admissible for the Fisher-KPP equation: These satisfy the relation $c \geq c_{m}$ where the minimal velocity $c_{m}$ plays a key role in describing the large time behaviour of solutions [56,57]. Furthermore, it has been shown that equations similar to Fisher-KPP above admit waves with variable velocity $[57,58]$.

In view of these facts, we suggest that a minimal model for the invasive process under consideration could consist of a three-component RD system for the concentrations $u, v$ and $w$ such that: (i) It exhibits pulse waves for $u$ and $w$, the wave pulse for $w$ actually preceding that of $u$, (ii) $v$ follows a Fisher-KPP propagating mechanism, (iii) The wave speeds agree with those experimentally measured by Alvarez et al. [47], and (iv) The wave speeds should undergo changes when passing from one invasive stage to the next one, in agreement with the behaviour recalled in figure 5 . These questions are currently being investigated.

\section{Secondary ossification}

\subsection{The dynamics of the process}

The beginning of SO is characterized by the formation of cartilage canals. These are invaginations originated in the surrounding perichondrium, which invade epiphyseal cartilage and produce intrachondrial canals by the fifth postnatal day [36,47]. Such invasion takes place at 3 or 4 , roughly equidistant, places located in the upper region of the epiphysis (cf. figure 8).

Canals proceed from the periphery to the centre, keeping an almost constant width, and moving at a relative high speed, so that they reach the central epiphyseal zone in about 2 to 3 days. As invasion proceeds towards the middle of the epiphysis, the cartilage located therein shows signs of incipient hypertrophy, and displays small foci of calcified matrix.

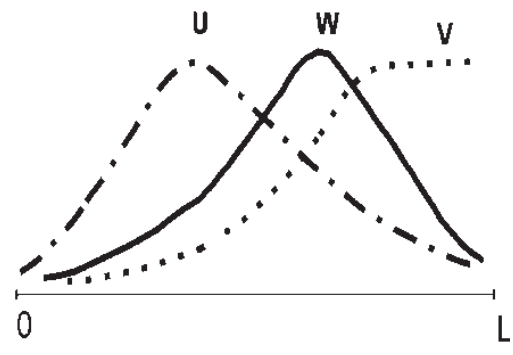

B

Figure 7. (A) Gradients of $u, v, w$ at $t=0$; (B) Subsequent evolution. A travelling wave behavior is proposed for that period. 

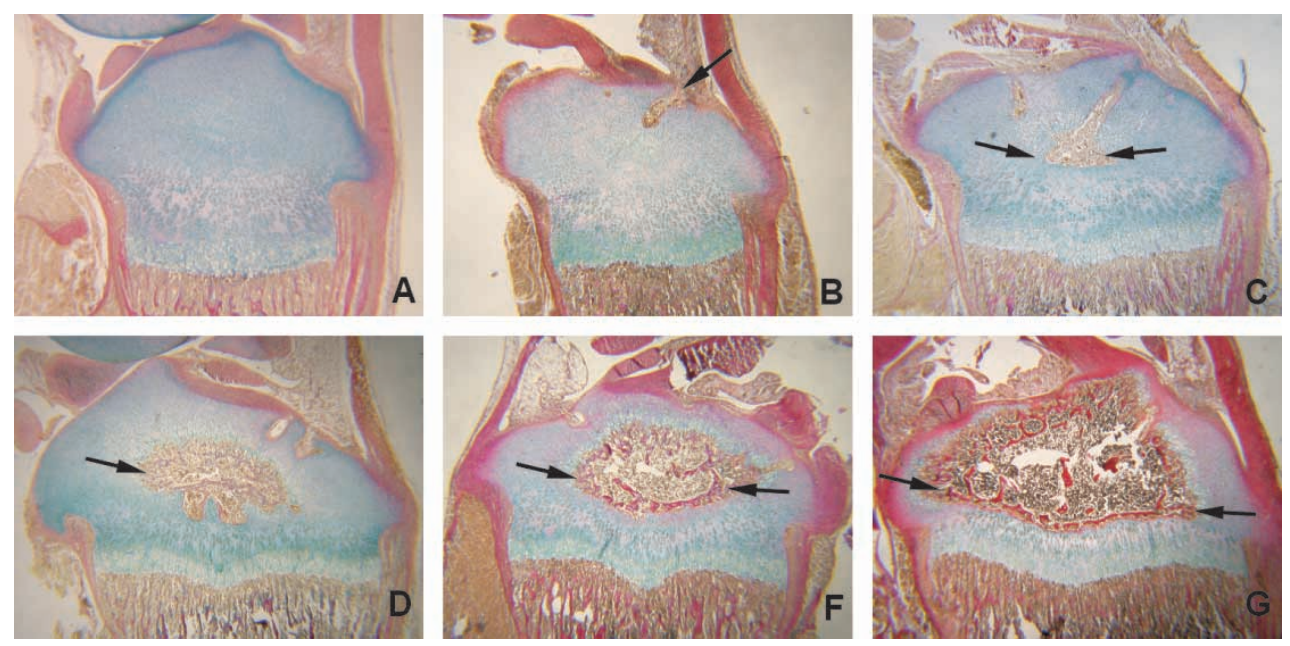

Figure 8. Progressive stages in ossification of the proximal epiphysis of the rat. The epiphysis is completely cartilaginous at the 3rd postnatal day (A). Cartilage canals appear at the 5th day (B, arrow). Canals reach the middle of the epiphysis and begin to spread transversely and fuse with each other to form a central dilated cavity which extends centrifugally $(\mathrm{C}-\mathrm{D}$, arrowheads). Invasion of the hypertrophic cartilage proceeds uniformly from the center to the periphery and this results in circumferential enlargement (D), but from day 11 onwards the ossification process becomes modified at the distal side of the epiphysis (that facing the metaphysis) and this divergence results in the establishment of a proximodistal polarity about the 14th postnatal day (F). From this point there is a progressive thinning of the epiphyseal cartilage and an increase of bony tissue $(\mathrm{G})$.

As soon as canals have reached the central zone, a second stage starts, in which these spread transversally and merge with each other to eventually produce a central cavity which subsequently enlarges centrifugally in an isotropic manner. In this way, a circular growth of this cavity is achieved, which goes hand in hand with increased cell proliferation at the interior and extended mineralization of the cartilaginous matrix. This stage lasts for 8 to 11 postnatal days in rat tibia, and results in the formation of a circular, secondary growth plate. The third stage that follows is the longest one (it extends from days 11 to 35 in the case under consideration), and is characterized by the fact that expansion in the distal zone of the cavity is arrested. Proximal and lateral zones continue to grow, however, so that the central cavity develops a hemispherical shape, which keeps on expanding in the (curved) proximal directions, but not along the straight, distal border. In analogy with the steady stage in primary ossification, both cartilage and bone keep on growing. However, once again bone expands at a slightly faster pace than cartilage, which results in a continuous depletion of cartilage being transformed in bone. By postnatal day 14, resting cartilage appears in most of the distal chondro-osseous junction.

Finally, a fourth stage extends from day 35 onwards, when a transverse bone trabecula is formed at the distal border. This lamellar bone provides a physical barrier between the SOC and the epiphyseal growing cartilage, and signals the end of cartilage resorption at the distal boundary (that is, that corresponding to bone expanding from the POC). Such process continues, however, at the proximal side, where a thin layer of articular cartilage eventually remains. This last plays a key role in the efficient performance of bone joints. SO thus yields the formation of convex epiphyses, which are suitable to obtain movable articulations with other bones. This final stage corresponds to the picture shown in figure 9 .

\subsection{Spatiotemporal patterns in SO}

Studies on bone development have been mainly concerned with primary ossification. Much less is known about the cellular processes involved in SO. Recently, the expression patterns corresponding to several MMPs, as well as to VGEF, in the course of secondary ossification in rat tibia have been analysed by Alvarez et al. [59]. These authors focused on three MMPs, namely gelatinase-B (MMP-9), collagenase-3 (MMP-13) and membrane-type 1 metalloproteinase (MMP-14), which were shown to exhibit different distribution patterns during SO. In particular, they showed that:

(1) The expression of MMP-9, MMP-13 and MMP-14 simultaneously starts at the perichondrial regions undergoing invagination, continues at the blind ends of advancing intrachondrial canals, and persists at the expanding borders of the central region. Expression of such MMP sharply decreases at locations where cartilage growth is halted.

(2) VGEF is not expressed during the formation of intrachondrial canals, but such expression occurs during formation of the central cavity. Passage from the second to the third stage described above (that is, from circular to hemispherical cavity shape) is strongly reflected in angiogenic activity. Indeed, VGEF is then shown to be highly expressed in proximal and lateral regions around the cavity, but significantly decreases along the distal side.

Secondary ossification presents significant differences with (and nevertheless should be coordinated to) primary ossification. When it comes to mathematical modelling, in this last case bone invasion can be accurately described by one-dimensional (in space) models, as those commented upon at the end of the former section. However, secondary 

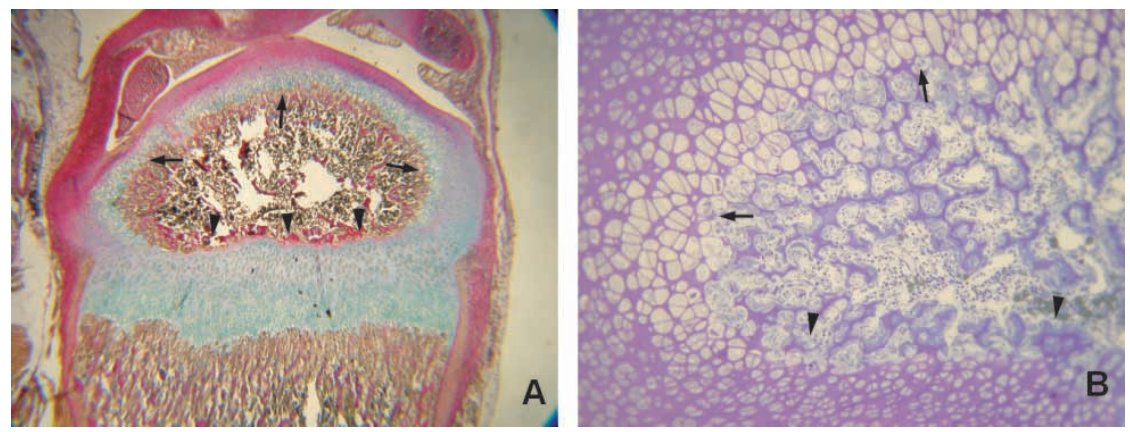

Figure 9. Secondary growth plate. Proximodistal polarity is evident in the epiphysis. Hypertrophic cartilage is only found at proximolateral sides (A, B arrows), whereas resting cartilage directly outlines the distal side (B, arrowheads). Epiphyseal cartilage at the proximal side presents many comparable aspects to that at the primary ossification center: Chondrocytes are organized in arched strata, with cells at resting, proliferating and hypertrophic stages arranged into radial columns (B).

ossification is essentially a higher-dimensional process, in which a spherically symmetric growth of the SOC is eventually turned into a hemispherical one, with both growth plates (primary and secondary) facing each other. We propose that, in agreement with the model previously postulated, a planar (essentially one-dimensional) and a spherical wave of VGEF are generated at the POC and SOC, respectively, and move towards each other, in the wake of similar inhibitory waves. When these meet each other, further expression of VGEF is avoided, and SOC keeps on growing only along the more distal zones from the anhilitation region. As a matter of fact, a related scenario, in which inhibitory zones are generated that preclude moving activated spots to coalesce, has been proposed in the context of blood coagulation dynamics (see Ataullakhanov et al. [60,61]). Again, deriving a minimal system for secondary ossification that fits the experimental findings of Alvarez et al. [59], is the subject of current investigation.

\section{Discussion and conclusions}

In this article we have reviewed some aspects of bone formation, starting from the early stages of embryonic development up to the formation, and mutual interaction, of primary and SOC in endochondral bones. Most of our discussion is particular to chick limb development (at the embryonic stage) and to rat tibia (when ossification develops), selected because of the wealth of experimental results available for such cases.

Besides presenting a short (and necessarily incomplete) overview on the biological studies conducted on these events, an attempt has been made to link known experimental facts with available mathematical methods, particularly suited to yield quantitative models of the processes involved. Such mathematical tools can provide relevant information in several ways. First, by allowing efficient numerical exploration for large sets of parameter data (which are generally difficult to derive experimentally), they could efficiently complement, and in some cases replace, laboratory experimentation. What is more, by rigorously exploring the (generally non-linear) interaction of the various agents involved, they permit a thorough analysis of putative signalling pathways, possibly pointing out redundancy when it occurs, uncovering the insufficiency of proposed interactions in some cases, and providing criteria to test robustness and modularity of the molecular networks under scrutiny.

The very nature of the examples just mentioned bear witness to the choice herein made of $\mathrm{RD}$ processes as the focus of this work. Indeed, these are thought to be essential at early development, and remain fundamental during the posterior life stages, since they correspond to the physical and chemical laws regulating signal trafficking. We are aware of the important role of mechanical aspects in cartilage and bone peformance, but we have decided not to deal with these types of topics here for definiteness. When describing some of the features of ossification, we have picked a number of examples as particularly suited for modelling in terms of RD equations. We have preferred to raise questions rather than focusing in the description of results already available for particular sets of equations. In any case, many of the standard tools of the trade in RD systems have been recalled in connection with biological processes. In particular, bistable and excitable systems, activator-inhibitor equations and wave propagation phenomena have been commented upon in the text, sometimes in the context of problems which are being currently investigated.

\section{Acknowledgements}

This work has been partially supported by European Contract MRTN-CT-2004-503661 and Spanish Ministry of Science Grant MCT-00-BMC-0446. The authors are grateful to Hans Meinhardt for a number of interesting discussions during the preparation of the manuscript.

\section{References}

[1] Wolpert, L., 1998, Principles of Development (Oxford University Press).

[2] Turing, A.M., 1952, The chemical basis of morphogenesis. Phil. Trans. R. Soc. Lond., 237, 37-72. 
[3] von Dassow, G., Meir, E., Munro, E.M. and Odell, G.M., 2000, The segment polarity network is a robust developmental module. Nature, 406, 188-192, 13 July.

[4] Raya, M., Kawakami, Y., Rodriguez-Esteban, C., Ibañes, M. Russkin-Gutman, D., Rodriguez-León, J., Buscher, D., Feijó, J.A. and Izpisúa-Belmonte, J.C., 2004, Notch activity acts as a sensor for extracellular calcium during vertebrate left-right determination. Nature, 427, 121-128.

[5] Carmeliet, P., 2000, Mechanisms of angiogenesis and arterogenesis. Nat. Med., 6(3), 389-395.

[6] Yancopoulos, G.D., Davis, S., Gale, N.W., Rudge, J.S., Wiegand, S.J. and Holash, J., 2000, Vascular-specific growth factors and blood vessel formation. Nature. 407, 242-248.

[7] Bussolino, F., Arese, M., Audero, E., Giraudo, E., Marchiò, S., Mittola, S., Primo, L. and Serini, G., 2003, Biological aspects of tumour angiogenesis. In: L. Preziosi (Ed.) Cancer Modeling and Simulation (Chapman and Hall), pp. 1-22.

[8] Sleeman, B.D. and Levine, H.A., 2001, Partial differential equations of chemotaxis and angiogenesis. Math. Meth. Appl. Sci., 24, 405-426.

[9] Levine, H.A., Sleeman, B.D. and Preziosi, L., 2003, Modelling tumour-induced angiogenesis. Cancer Modeling and Simulation (Chapman and Hall), pp. 147-184.

[10] Chaplain, M.A.J. and Anderson, A.R.A., 2003, Mathematical modeling of tissue invasion. In: L. Preziosi (Ed.) Cancer Modeling and Simulation (Chapman and Hall), pp. 269-298.

[11] Gardiner, D.M., Blumberg, B., Komine, Y. and Bryant, B.V., 1995, Regulation of HoxA expression in developing and regenerating axolotl limbs. Development, June 121(6), 1731-1741.

[12] Brand-Saberi, B. and Christ, B., 2000, Evolution and development of distinct cell lineages derived from somites. Curr. Top. Dev. Biol., 48, $1-42$.

[13] Capdevila, J. and Izpisúa-Belmonte, J.C., 2001, Patterning mechanisms controlling vertebrate limb development. Annu. Rev. Cell. Dev. Biol., 17, 87-132.

[14] Meinhardt, H., 1982, Models of Biological Pattern Formation (London: Academic Press).

[15] Meinhardt, H., 1983a, A boundary model for pattern formation in vertebrate limbs. J. Embryol. Exp. Morph., 76, 115-137.

[16] Maden, M., 1983, A test of the predictions of the boundary model regarding supernumerary limb structures. J. Embryol. Exp. Morph. 76, $147-155$

[17] Meinhardt, H., 2004, Different strategies for midline formation in bilaterians. Nat. Rev. Neurosci., 5, 502-509.

[18] Gierer, A. and Meinhardt, H., 1972, A theory of biological pattern formation. Kybernetik, 12, 30-39.

[19] Koch, A.J. and Meinhardt, H., 1994, Biological pattern formation: From basic mechanisms to complex structures. Rev. Mod. Phys., 66(4), 1481-1507.

[20] de Lise, A.M., Fischer, L. and Tuan, R.S., 2000, Cellular interactions and signaling in cartilage development. Osteoarthritis Cartilage, 8, 309-334.

[21] Rowe, D.A. and Fallon, J.F., 1982, Normal anterior pattern formation after barrier placement in the chick leg: Further evidence on the action of the polarizing zone. J. Embryol. Exp. Morph., 69, $1-6$.

[22] Summerbell, D., 1974, A quantitative analysis of the effect of excision of the AER from the chick limb-bud. J. Embryol. Exp. Morph., 32, 651-660.

[23] Summerbell, D., 1978, Normal and experimental variations in preparations of skeleton of chick embryo wing. Nature, 274(5670), $472-473$.

[24] Wolpert, L., 2002, The progress zone model for specifying positional information. Int. J. Dev. Biol., 46(7), 869-870.

[25] Ng, J.K., Tamura, K., Buscher, D. and Izpisúa-Belmonte, J.C., 1999, Molecular and cellular basis of pattern formation during vertebrate limb development. Curr. Top. Dev. Biol., 41, 37-66.

[26] Mercader, N., Leonardo, E., Azpiazu, N., Serrano, A., Morata, G., Martinez, C. and Torres, M., 1999, Conserved regulation of proximodistal limb axis development by Meis1/Htb. Nature, 402(6760), 425-429.

[27] Meinhardt, H., 1983b, A bootstrap model for the proximodistal pattern formation in vertebrate limbs. J. Embryol. Exp. Morph., 76 $139-146$.

[28] Maden, M. and Mustafa, K., 1982, The structure of 180 degrees supernumerary limbs and a hypothesis of their formation. Dev Biol., 93(19), 257-265.
[29] Tickle, C., Alberts, B., Wolpert, L. and Lee, J., 1982, Local application of retinoic acid to the limb bud mimics the action of the polarizing region. Nature, 296(5857), 564-566.

[30] Summerbell, D., 1983, The effect of the application of retinoic acid to the anterior margin of the developing chick limb. J. Embryol. Exp. Morph., 78, 269-289.

[31] Thaller, C. and Eichele, G., 1996, Retinoid signaling in vertebrate limb development. Ann. N Y Acad. Sci., 785, 1-11.

[32] Mic, F.A., Sirbu, I.O. and Duester, G., 2004, Retinoic acid synthesis controlled by Raldh 2 is required early for limb bud initiation and then later as a proximodistal signal during apical ectodermal ridge formation. J. Biol. Chem., 279(25), 26698-26706.

[33] Dillon, R. and Othmer, H.G., 1999, A mathematical model for outgrowth and spatial patterning of the vertebrate limb and bud J. Theor. Biol., 197, pp 295-330.

[34] Gadgil, C., Dillon, R., and Othmer, H.G., 2003, Short and longrange efects of Sonic hedgehog in limb development. Proc. Nat. Acad. Sci. USA, 100, pp 819-835.

[35] Hall, B.K. and Miyake, T., 2000, All for one and one for all: condensations and the initiation of skeletal development. BioEssays, 22, 138-147.

[36] Rivas, R. and Shapiro, F., 2002, Structural stages in the development of the long bones and epiphysis. J. Bone Joint Surg. 84(1), 85-100.

[37] Keller, E.F. and Segel, L.A., 1970, Initiation of slime mold aggregation viewed as an instability. J. Theor. Biol., 26, 399-415.

[38] Gomer, R., Gao, T., Tang, Y., Knecht, D. and Titus, M.A., 2002, Cell motility mediates tissue size regulation in Dictyostelium. J. Muscle Res. Cell Motil., 23, 809-815.

[39] Escalante, R. and Vicente, J.J., 2000, Dictyostelium discoideum: a model system for differentiation and patterning. Int. J. Dev. Biol., 44, pp 819-835.

[40] Herrero, M.A., 2003, Reaction-diffusion systems: A mathematical biology approach. In: L. Preziosi (Ed.) Cancer Modeling and Simulation (Chapman and Hall), pp. 367-420.

[41] Horstmann, D., 2003, From 1970 until present: The Keller-Segel model in chemotaxis and its consequences I. Jahresbericht der DMV, 105(3), 103-165.

[42] Herrero, M.A. and Velázquez, J.J.L., 1996, Chemotactic collapse for the Keller-Segel model. J. Math. Biol., 75, 177-196.

[43] Velázquez, J.J.L., 2002, Stability of some mechanisms of chemotactic aggregation. SIAM J. Appl. Math., 62, 1581-1633.

[44] Hentschel, H.G.E., Glimm, T., Glazier, J.A. and Newman, S.A., 2004, Dynamical mechanisms for skeletal pattern formation in the vertebrate limb. Proc. R. Soc. Lond. B, 271(1549), 1713-1722.

[45] Hunziker, E.B., 1994, Mechanism of longitudinal bone growth and its regulation by growth plate chondrocytes. Microsc. Res. Tech., 28 $505-519$.

[46] Kronenberg, H.M., 2003, Developmental regulation of the growth plate. Nature, 423, 332-336.

[47] Alvarez, J., Balbín, M., Santos, F., Fernández, M., Ferrando, S. and López, J.M., 2000, Different growth rates are associated with changes in the expression pattern of types II and X collagens and collagenase 3 in proximal growth plates of the rat tibia. J. Bone. Miner. Res., 15(1), 82-94.

[48] Roach, H.I., Baker, J.E. and Clarke, N.M.P., 1998, Initiation of bone epiphysis in long bones: Chronology of interactions between the vascular system and the chondrocytes. J. Bone Miner. Res., 13(6), 950-961.

[49] Cancedda, R., Descalzi Cancedda, F. and Castagnola, P., 1995, Chondrocyte differentiation. Int. Rev. Cytol., 159, 265-358.

[50] Olsen, B.R., Reginato, A.M. and Wenfang, W., 2000, Bone development. Ann. Rev. Cell Dev. Biol., 16, 191-220.

[51] Mikhailov, A.S., 1994, Foundations of Synergetics I (SpringerVerlag).

[52] Murray, J.D., 2000, Mathematical Biology II, Springer Interdisciplinary Applied Mathematics, Vol. 18.

[53] Fisher, R.A., 1937, The wave of advance of an advantageous gene. Ann. Eugen., 7, 353-369.

[54] Kolmogorov, A. Petrovsky, I. and Piskunov, N., 1988. Study of the diffusion equation with growth of the quantity of matter and its application to a biology problem. In: P. Pelcé (Ed.) (Trans.) (London: Academic Press) (Original work published 1937, Bul Moskovskovo G.S. Univ. 17, 1-26).

[55] Grindrod, P., 1991, Patterns and Waves (Oxford: Oxford University Press). 
[56] Larson, D.A., 1978, Transient bounds and time-asymptotic behaviour of solutions to nonlinear equations of Fisher type. SIAM J. Appl. Math., 34, 93-103.

[57] Needham, D.J. and Barnes, A.N., 1999, Reaction-diffusion and phase waves occurring in a class of scalar reaction-diffusion equations. Nonlinearity, 12, 41-58.

[58] Kay, A.L., Sherratt, J.A. and Mc Leod, J.B., 2001, Comparison theorems and variable speed waves for a scalar reaction-diffusion equation. Proc. R. Soc. Edinb. A, 131, 1133-1161.

[59] Alvarez, J., Costales, L., Serra, R., Balbín, M. and Lopez, J.M., 2005, Expression patterns of matrix metalloproteinases and vascular endothelium growth factor during epiphyseal ossification. J. Bone. Miner. Res., To appear.

[60] Ataullakhanov, F.I., Guria, G.T., Sarbash, V.I. and Volkova, R.I., 1998, Spatio-temporal dynamics of clotting and pattern formation in human blood. Biochim. Biophys. Acta, 1425, 453-468.

[61] Ataullakhanov, F.I., Krasotkina, V., Sarbash, V.I., Volkova, R.I., Sinavridse, E.I. and Kondratovich, A.Y., 2002, Spatiotemporal dynamics of blood coagulation and pattern formation: An experimental study. Int. J. Bifurcat. Chaos, 12(9), $1969-1983$. 


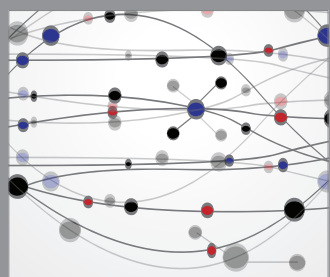

The Scientific World Journal
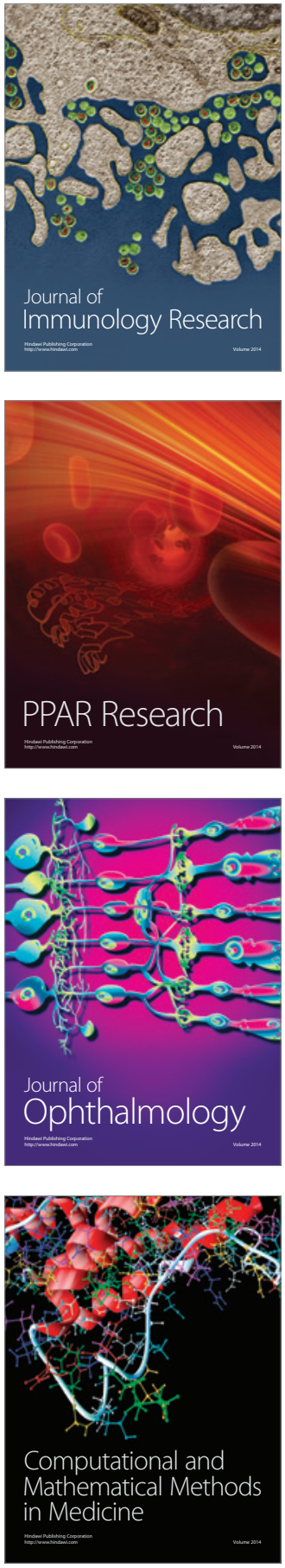

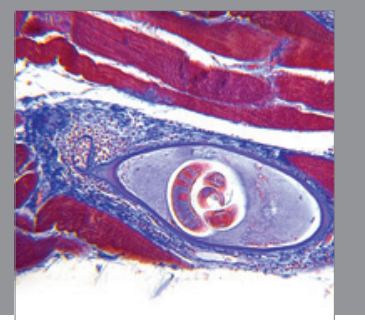

Gastroenterology

Research and Practice
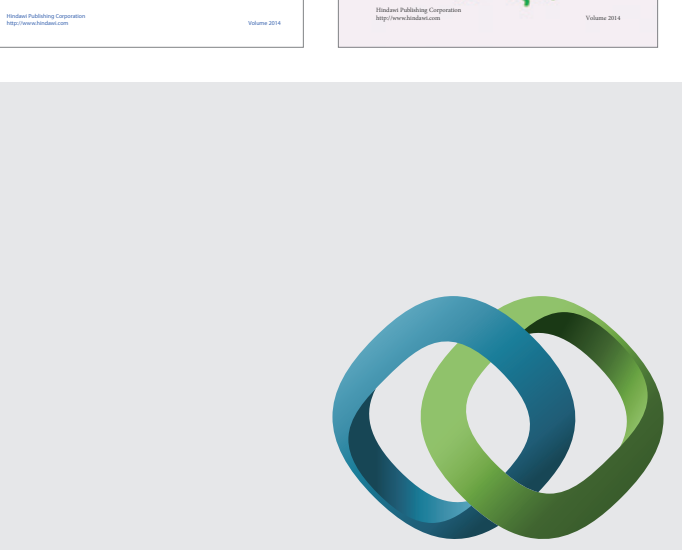

\section{Hindawi}

Submit your manuscripts at

http://www.hindawi.com
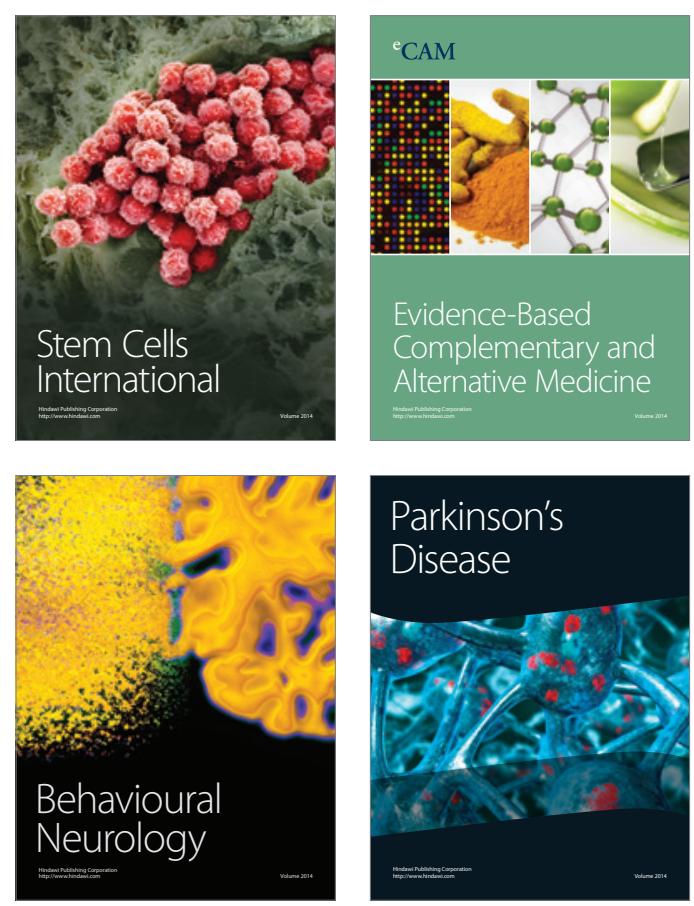

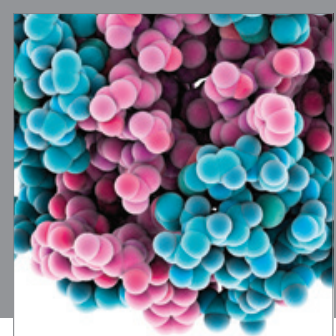

Journal of
Diabetes Research

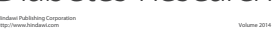

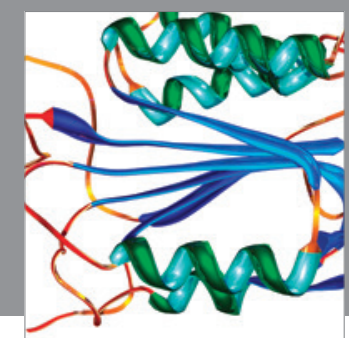

Disease Markers
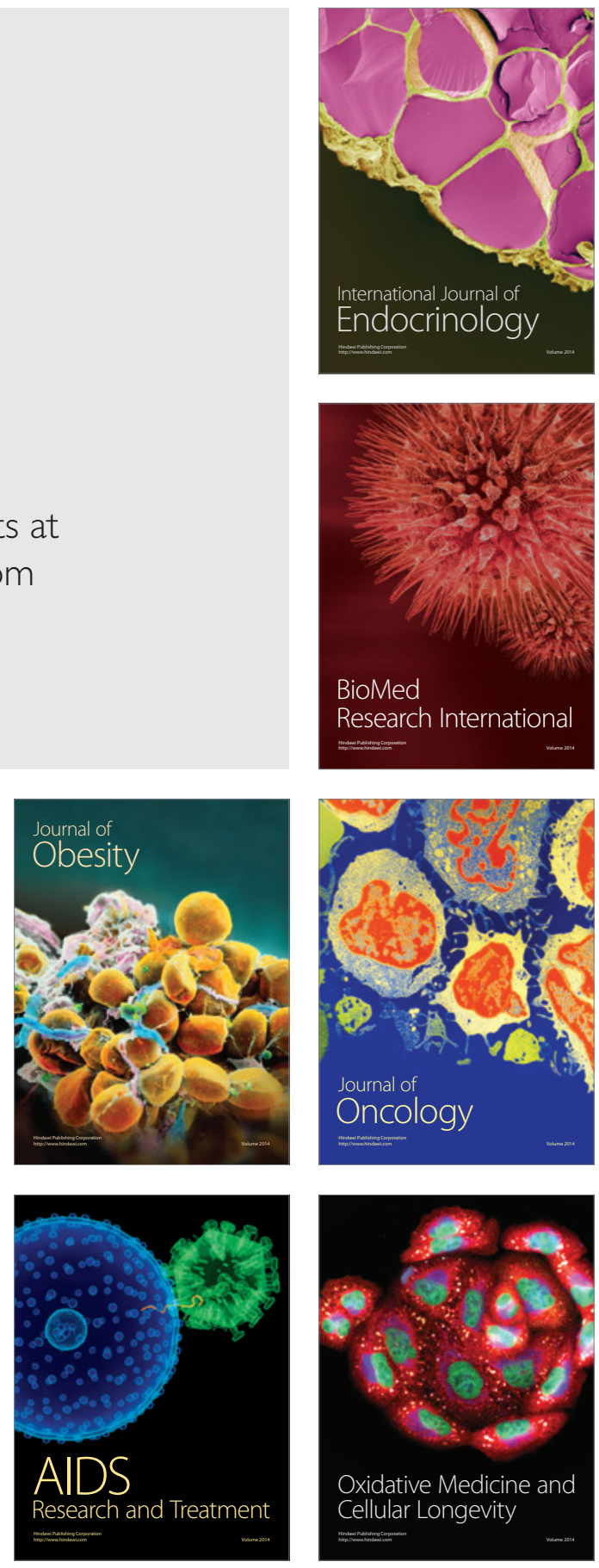